\title{
Aptitude des vaches à la traite mécanique : relations avec certaines caractéristiques physiques du trayon
}

\author{
J Le Du 1, FA de La Chevalerie 1, M Taverna 2, Y Dano 1 \\ 1 INRA, laboratoire de recherches sur la traite, 65, rue de Saint-Brieuc, 35042 Rennes, France; \\ 2 INTA, station d'expérimentation agronomique, 2300 Rafaela, Argentine
}

(Reçu le 13 avril 1992; accepté le 29 mars 1993)

\begin{abstract}
Résumé - Les relations entre certaines caractéristiques physiques du trayon et l'aptitude à la traite sont étudiées sur 30 vaches de race Française Frisonne Pie Noire. La cinétique d'écoulement du lait pendant la traite est enregistrée séparément pour chaque quartier. Les propriétés élastiques de l'extrémité du trayon sont mesurées en appliquant des pressions et des succions sur les tissus à l'aide de divers appareils d'une conception simple. Les quartiers arrière ont des temps de traite et des débits d'écoulement du lait plus élevés que l'avant $(P<0,001)$. Cela provient du déséquilibre antéropostérieur car l'arrière produit $60 \%$ du lait. Mais il apparaît aussi que les trayons arrière se traient plus facilement que ceux de l'avant. À l'arrière, la pression ou la succion qui permet l'ouverture du canal du trayon et un début d'écoulement du lait est significativement plus faible qu'à l'avant. En outre, la section du canal du trayon est plus extensible $(P<0,001)$. Les trayons les plus faciles à traire ont une paroi peu épaisse avec un canal terminal entouré d'un faible volume de tissus élastiques. II en résulte une diminution des temps de traite et un accroissement des débits. Le débit maximal en particulier est bien corrélé avec la quantité de lait recueillie en appliquant pendant $20 \mathrm{~s}$ un vide constant sous le trayon à l'aide du vacuomètre $(r=+0,75)$. Cet équipement, facile à utiliser en fermes, pourrait permettre au généticien d'apprécier rapidement l'aptitude à la traite (20 à 30 vaches par heure), indépendamment des effets du milieu. Les modalités d'emploi du vacuomètre demanderaient à être précisées.
\end{abstract}

vache laitière / trayon / aptitude à la traite / traite / mamelle

Summary - Milkability of cows: relationships with certain teat characteristics. Relationships between certain teat characteristics and milkability were studied in 30 French Friesian black and white cows. Milking characteristics were recorded separately for each quarter. Using relatively simple equipment, pressures and suctions of several types were applied in order to determine certain elastic properties of the teat tip. Milking times and flow rates were higher for the hindquarters than for the forequarters $(\mathrm{P}<0.001)$. This was due to the antero-posterior disequilibrium as $60 \%$ of the milk was stored in the hindquarters. In addition, some results showed that rear-teats were easier to milk than fore-teats. The vacuum and pressure required to open the teat canal and observe the beginning of milk flow were lower than for front teats. The stretchability of the teat canals was also higher $(\mathbb{P}<0.001)$. Teats that were easy to milk had thin walls and a teat canal surrounded by a 
small volume of elastic tissue. Consequently, milking times were decreased and milk flow rates increased. Using experimental equipment (ie the vacuometer), the correlation between the maximum milk flow rate and the milk volume extracted when a constant vacuum was maintained for $20 \mathrm{~s}$ beneath the teat reached +0.75 . Consequently, use of the vacuometer might provide a practical testing method for assessment of milk flow rate. The equipment was easy to handle under farm conditions and the measurements were made rapidly $(20-30 \mathrm{cows} / \mathrm{h})$.

dairy cow / teat / milkability / milking / udder

\section{INTRODUCTION}

L'écoulement du lait pendant la traite est influencé par les caractéristiques physiques du trayon. On considère que la section du canal est un facteur prépondérant (Baxter et al, 1950). Les mesures effectuées à l'aide de sondes coniques calibrées (Johansson, 1957 ; Johansson et Malven, 1960 ; Naude et Smith, 1964) ou par introduction de branches expansibles (Andreae, 1958 ; Labussière, 1972), ainsi que la radiographie aux rayons $X$ (Andreae, 1958 ; McDonald, 1968a, b, 1975), indiquent que la section du canal du trayon est corrélée positivement avec le débit d'écoulement du lait et négativement avec le temps de traite.

Les observations effectuées après abattage des animaux ne permettent pas de confirmer cette dépendance mais montrent que les vaches faciles à traire auraient des trayons avec des parois minces et un canal court entouré d'un tissu musculaire peu abondant par rapport au tissu conjonctif (Kuhne, 1959 ; Loppnow, 1959 ; Barczynski, 1960). Ces conclusions semblent valider l'étude théorique de Balthazar et Scott (1977) et les résultats expérimentaux de Schmitten et Klüsserath (1968), Rathore (1977), Rathore et Sheldrake (1977) qui considèrent que le débit du lait est plus élevé pour les trayons de grands diamètres à parois minces. Toutefois, d'autres travaux font état d'une corrélation négative (Skallcup et al, 1963 ; Johansson et Malven, 1960 ; Sandvik, 1957) ou non significative (Naude et Smith, 1964 ; Ovesen, 1970, 1972 ; Notsuki et Goto, 1967b ; Naito et al, 1965b ; Wilke, 1959) entre le débit du lait et le diamètre du trayon. II n'est donc pas possible d'envisager l'utilisation de critères aussi simples pour évaluer l'aptitude à la traite.

En fait, durant la traite, pour provoquer l'ouverture du canal du trayon, il est nécessaire d'induire des contraintes relativement élevées dans les tissus (Towsend, 1969 ; Balthazar et Scott, 1977). L'écoulement du lait n'est pas conditionné seulement par la section et la longueur du canal. En théorie, la forme du canal, la texture de ses parois et la pression intramammaire auraient une influence (Karavaev, 1966; Valdmann, 1959 ; Notsuki et Goto, 1967b ; Johansson et Malven, 1960; Mein et Williams, 1984 ; Williams et Mein, 1982). II en résulte qu'en mettant les tissus sous contrainte, la force de succion (Notsuki et Goto, 1967a ; Kartashov, 1973 ; Zhukov, 1971) ou de compression (Notsuki et Goto, 1967a ; Naito et al, 1965a ; Zhukov, 1971) à appliquer au trayon pour obtenir un début d'écoulement du lait apparaît être un bon critère d'appréciation de l'aptitude à la traite. Selon Notsuki et Goto (1967b), la corrélation avec le débit maximal (succion : $r=-0,81$; compression : $r=$ $-0,71$ ) serait similaire à celle obtenue en mesurant directement le diamètre du canal $(r=0,85)$. Naito et al (1965b) estiment même que le tonus du trayon par compression ( $r=-0,67$ et $r=-0,72)$ 
convient mieux que le diamètre du canal du trayon $(r=0,32)$ pour apprécier le débit maximal.

Pour l'amélioration génétique, les mesures de ce type sont susceptibles de fournir des critères d'appréciation de l'aptitude à la traite plus représentatifs que ceux qui sont obtenus par les méthodes actuelles de chronométrage du temps de traite (Colleau, 1971 ; Krychowski, 1972). En effet, dans ce dernier cas, l'habileté du trayeur, les caractéristiques de l'installation de traite et les conditions d'élevage constituent des facteurs de variation qui perturbent le jugement porté sur l'aptitude laitière intrinsèque des filles ou des mères à taureau.

Dans ce contexte, nous présentons ici une étude des relations existant entre les caractéristiques de traite des vaches et certaines propriétés physiques de l'extrémité du trayon évaluées au moyen d'appareils susceptibles d'être utilisables à la ferme.

\section{MATÉRIEL ET MÉTHODES}

\section{Mesure des caractéristiques des trayons}

Les mesures sont réalisées après massage de la mamelle et extraction des premiers jets de lait avant la traite.

\section{Duromètre (fig 1)}

Le duromètre est un compas d'épaisseur à ressort qui permet d'appliquer une pression sur le trayon en le comprimant entre 2 disques de 30 mm de diamètre (Le Du et Taverna, 1989 ; Hamann et Mein, 1990 ; Zecconi et al, 1992). Les mesures effectuées sur le trayon avant la traite sont les suivantes:

- le diamètre nominal de l'extrémité ( $\mathrm{mm}$ ) est mesuré sans aucune compression du trayon :
- l'épaisseur de l'extrémité (mm) est mesurée avec une force de compression de $2,4 \mathrm{~kg}$ appliquée à l'extrémité du trayon (fig 1) ;

- l'épaisseur des parois est mesurée avec une force de $2,4 \mathrm{~kg}$ appliquée dans la partie médiane du trayon.

\section{Garrot (fig 2)}

Le garrot est composé d'un cylindre rigide de 3 $\mathrm{cm}$ de hauteur (a). Un tube en caoutchouc (b) est fixé à l'intérieur de ce cylindre de façon à créer une chambre annulaire. Après clampage du trayon, la pression dans cet espace annulaire est progressivement augmentée. Le tonus du trayon est la pression à appliquer $(\mathrm{kPa})$ pour obtenir un début d'écoulement du lait.

\section{Vacuomètre (fig 3)}

Le vacuomètre se compose d'un gobelet rigide transparent relié à une pompe à vide qui permet de créer une dépression sous le trayon inséré dans le gobelet. Une cuve intercalée entre le gobelet et la pompe permet de recueillir le lait extrait. Une admission d'air réglable permet de faire varier le niveau de vide.

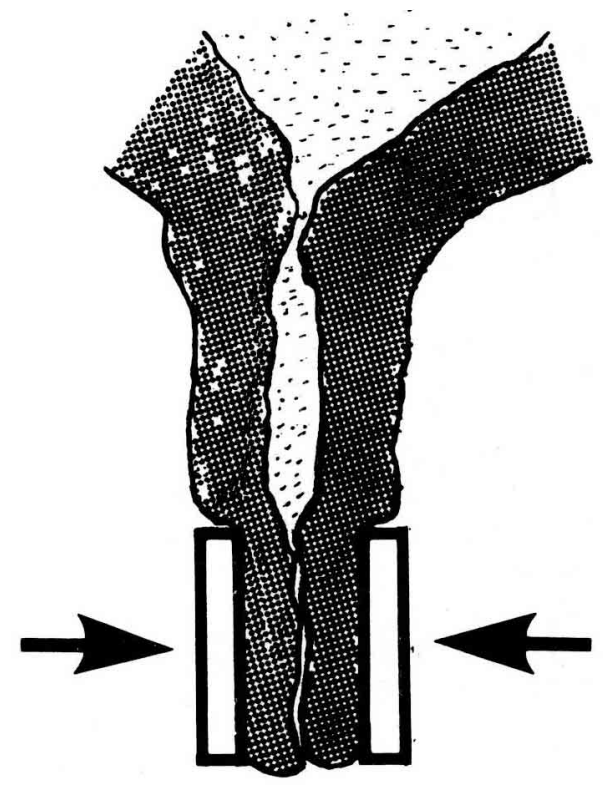

Fig 1. Compas d'épaisseur à ressort. 
Les mesures effectuées avec le vacuomètre sont les suivantes :

- le tonus du trayon par succion est mesuré en appliquant un vide croissant sous le trayon à la vitesse de $1 \mathrm{kPa} / \mathrm{s}$. Le vide ( $\mathrm{kPa}$ ) correspondant à l'écoulement des premières gouttes de lait représente la résistance du canal du trayon à s'ouvrir sous l'effet de la succion;

- la quantité de lait recueillie en $20 \mathrm{~s} \mathrm{(ml)} \mathrm{repré-}$ sente une autre évaluation des possibilités d'ex-

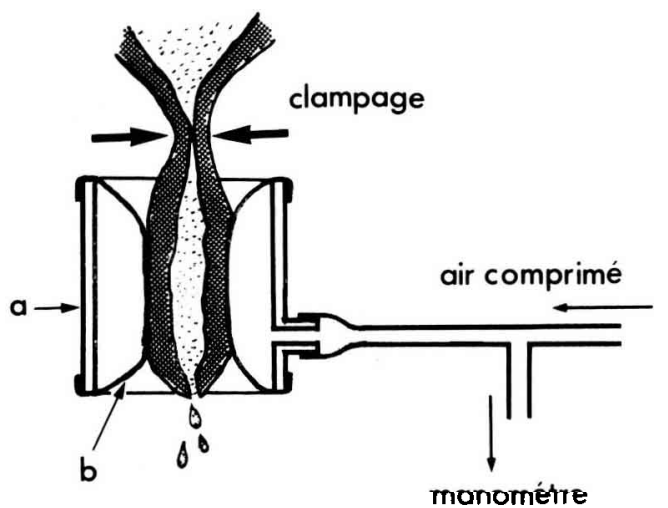

Fig 2. Garrot.

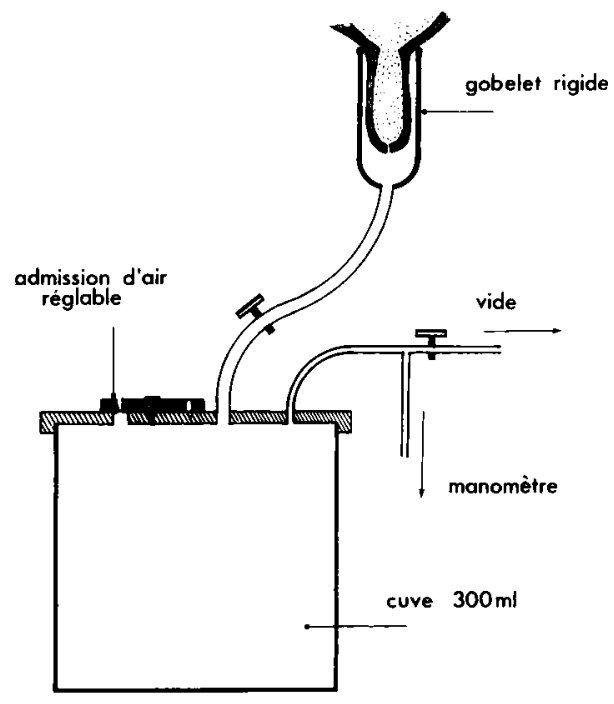

Fig 3. Vacuomètre. tension du canal du trayon. Elle est mesurée en appliquant un vide constant de $48 \mathrm{kPa}$ sous le trayon pendant $20 \mathrm{~s}$.

\section{Enregistrement des caractéristiques de traite des vaches}

L'enregistrement de la cinétique d'émission du lait par quartier (Labussière et Martinet, 1964) permet de définir les paramètres suivants :

- lait total : volume total de lait recueilli, égal à la somme du lait machine et du lait d'égouttage (en I) par quartier ;

- lait machine : volume total de lait extrait par la machine seule, avant intervention du trayeur (en 1), par quartier ;

- lait d'égouttage : volume du lait obtenu lors de l'opération d'égouttage pratiquée par le trayeur (I), par quartier ;

- temps total de traite : temps qui s'écoule entre la pose et la dépose des gobelets (en s) ;

- temps d'écoulement du lait machine : temps qui sépare la pose des gobelets du moment où le débit du lait, par quartier, devient $<0,050$ ।/ $\min (e n s)$;

- débit maximal : volume de lait maximal recueilli durant 2 intervalles consécutifs de $6 \mathrm{~s}$ chacun (en $\mathrm{l} / \mathrm{min}$ ) par quartier ;

- débit moyen : volume de lait machine divisé par le temps d'écoulement du lait machine (en I/ min), par quartier.

En outre, la facilité de traite des vaches est appréciée par la moyenne des notes attribuées indépendamment par chacun des 5 trayeurs selon la notation suivante : 20 , facile à traire ; 10 , normale ; 0 , longue à traire.

La traite s'effectue, dans des stalles en tandem, en ligne basse avec un niveau de vide de $50 \mathrm{kPa}$, une vitesse de pulsation de $60 \mathrm{p} / \mathrm{min}$ et un rapport de pulsation de $72 \%$. La manchon est du type 960 000-01 de marque Alfa-Laval.

\section{Protocole expérimental}

Les mesures des caractéristiques du trayon sont effectuées sur 30 vaches de race Française Frisonne Pie Noire, un matin et un soir sur les trayons droits de l'avant et de l'arrière, sans 
répétitions. La cinétique de descente du lait est enregistrée à une traite du matin et à une traite du soir. La traite s'effectue à $7 \mathrm{~h} 00$ le matin et à 16 h 00 le soir (intervalles de 9 et 15 h).

La répartition des animaux en fonction du numéro de lactation est la suivante : 5 vaches en

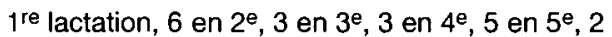
en $6^{e}, 2$ en $7^{e}, 2$ en $8^{e}, 1$ en $9^{e}$ et 1 en $10^{e}$. Au début de l'expérimentation, les stades de lactation sont distribués de façon presque régulière entre 66 et 240 jours ; la moitié des animaux ayant plus de 150 jours de lactation.

\section{RÉSULTATS ET DISCUSSION}

\section{Comparaison des caractéristiques de traite des trayons avant et arrière, la matin et le soir (tableau I)}

Les mamelles des animaux sont très déséquilibrées puisque le lait des quartiers avant représente $39,3 \%$ de la production.
Or, dans sa revue bibliographique, Szeremeta (1987) mentionne des indices antéropostérieurs moyens variant entre 39,1 et $47,0 \%$ pour les grandes races laitières. Cela explique probablement que tous les paramètres de traite (productions de lait, temps et débits d'écoulement du lait) sont significativement plus élevés pour les trayons arrière $(P<0,001)$. En effet, on admet que le débit augmente avec la production mais que l'accroissement du débit est insuffisant pour compenser l'effet d'une augmentation de la production. Le temps de traite est donc plus long lorsque la production s'accroît (Dodd et Griffin, 1979).

II est également possible que certaines différences soient liées à l'anatomie du trayon. Ainsi, le diamètre des trayons arrière, plus élevé qu'à l'avant $(P<0,001)$, pourrait expliquer l'accroissement des débits $(P<0,001)$ selon les hypothèses formulées par Balthazar et Scott (1977). Les caractéristiques du canal du trayon ont

Tableau l. Comparaison des caractéristiques de traite et des propriétés physiques des trayons : différences entre l'avant et l'arrière de la mamelle. Effets des intervalles entre traites (9 et $15 \mathrm{~h}$ ).

\begin{tabular}{|c|c|c|c|c|c|c|}
\hline & \multicolumn{2}{|c|}{ Trayons } & \multirow{2}{*}{$\begin{array}{c}\text { Valeurs } \\
\text { det }\end{array}$} & \multicolumn{2}{|c|}{ Horaires } & \multirow{2}{*}{$\begin{array}{c}\text { Valeurs } \\
\text { de } \mathrm{t}\end{array}$} \\
\hline & Avant & Arrière & & Matin & Soir & \\
\hline Lait total (l) & 1,88 & 2,90 & $10,30^{* * *}$ & 3,07 & 1,72 & $19,00^{* * *}$ \\
\hline Lait machine (I) & 1,77 & 2,71 & $8,90^{\star \star \star}$ & 2,91 & 1,57 & $16,00^{* * *}$ \\
\hline Lait égouttage (ml) & 105,0 & 181,0 & $4,40^{\star \star \star}$ & 143,0 & 143,0 & $0,00 n s$ \\
\hline Débit moyen ( $(1 / \mathrm{min})$ & 0,52 & 0,69 & $7,50^{\star \star \star}$ & 0,66 & 0,54 & $8,10^{\star \star \star}$ \\
\hline Débit maximal $(1 / \mathrm{min})$ & 0,78 & 0,94 & $4,40^{* * *}$ & 0,90 & 0,82 & $3,70^{* * *}$ \\
\hline Temps total (s) & 275,6 & 305,8 & $4,20^{\star \star \star}$ & 339,3 & 242,1 & $12,20^{\star \star \star}$ \\
\hline Temps machine (s) & 208,9 & 238,7 & $4,80^{*+*}$ & 271,1 & 176,5 & $12,90^{* * *}$ \\
\hline \multicolumn{7}{|l|}{ Diamètre nominal } \\
\hline extrémité trayon $(\mathrm{mm})$ & 23,76 & 25,06 & $5,40^{\star \star \star}$ & 24,89 & 23,93 & $4,10^{\star \star \star}$ \\
\hline Épaisseur extrémité trayon (mm) & 9,20 & 9,30 & 1,10 ns & 9,20 & 9,30 & $1,20 n s$ \\
\hline Épaisseur des parois (mm) & 8,06 & 8,09 & $0,00 n s$ & 8,07 & 8,07 & $0,00 \mathrm{~ns}$ \\
\hline Vide écoulement du lait ( $\mathrm{kPa}$ ) & 17,0 & 14,5 & $2,30^{*}$ & 15,3 & 16,2 & $1,10 n s$ \\
\hline Lait en $20 \mathrm{~s}(\mathrm{ml})$ & 110,0 & 158,8 & $3,80^{\star \star \star}$ & 146,6 & 122,2 & $3,30^{\star \star}$ \\
\hline Pression écoulement du lait $(\mathrm{kPa})$ & 17,2 & 10,9 & $4,70^{\star \star \star}$ & 13,7 & 14,3 & $0,10 n s$ \\
\hline
\end{tabular}

Les différences sont significatives aux seuils de ${ }^{* *}: 0,001(t=3,460) ;{ }^{* \star}: 0,01(t=2,660) ;{ }^{*}: 0,05(t=2,000)$ et $n s$ : non significatives au seuil $P=0,05$ pour 59 degrés de liberté (tests $t$ par valeurs couplées). 
certainement une incidence puisque, dans 2 races de vaches, Rathore et Sheldrake (1977) observent que l'élasticité du canal du trayon est plus élevée à l'arrière qu'à l'avant en étant corrélée positivement avec le diamètre extérieur du trayon $(P<0,01)$. En revanche, les différences éventuelles des pressions intramammaires entre l'avant et l'arrière ne semblent pas susceptibles d'avoir un effet important (Williams et Mein, 1982).

A une exception près (volume d'égouttage) le matin, les paramètres de traite ont des valeurs significativement plus élevées que le soir $(P<0,001)$. Compte tenu des intervalles très inégaux entre traites ( 9 et 15 h), cet effet provient également d'un déséquilibre des productions laitières puisque, le soir, on recueille seulement $36 \%$ du lait.

Le volume d'égouttage, exprimé en pourcentage de la production, est moins élevé le matin $(4,5 \%)$ que le soir $(7,7 \%)$. Avec des conditions de traite similaires, quel que soit le faisceau-trayeur, tous les travaux antérieurs (par exemple Le Du et Richard, 1977 ; O'Shea et O'Callaghan, 1980) confirment ce résultat mais montrent qu'il n'y a pas de différences marquées entre le matin et le soir lorsque les volumes d'égouttage sont exprimés en valeurs absolues.

\section{Comparaison des caractéristiques physiques des trayons à l'avant et à l'arrière, le matin et le soir (tableau I)}

Il existe peu de différences significatives.

Le diamètre du trayon est plus élevé à l'arrière qu'à l'avant $(P<0,001)$, comme l'observent également Notsuki et Goto (1967a) $(P<0,01)$. Cependant, il arrive que les trayons avant soient légèrement plus gros que les trayons arrière puisque Naito et al (1965b) citent un troupeau où le rapport avant-arrière est de 1,02.
Le matin, les trayons ont en moyenne un diamètre plus élevé que le soir $(P<$ 0,001 ), bien que l'épaisseur des tissus demeure constante. Le fait bien connu des trayeurs a été notamment rapporté par Benmederbel (1980) qui a observé sur notre troupeau que le diamètre du trayon augmente de façon continue pour des intervalles croissants entre traites de 4, 8, 12,16 et $20 \mathrm{~h}$. II est la conséquence directe d'un accroissement de la pression intramammaire provoqué par l'accumulation du lait dans la mamelle (Dodd et Griffin, 1979).

Les observations précédentes montrant que tous les paramètres caractérisant la cinétique d'écoulement du lait (temps et débits) sont plus élevés pour les trayons arrière s'interprètent logiquement en examinant les propriétés physiques que nous avons mesurées. Globalement, nous constatons, comme Rathore et Sheldrake (1977), mais avec d'autres critères, que les trayons arrière se traient plus facilement que ceux de l'avant. La succion provoquant le premier écoulement du lait est plus faible : 14,5 contre $17,0 \mathrm{kPa}(P<$ $0,05)$. La quantité de lait recueilli en $20 \mathrm{~s}$ sous succion constante est plus élevée de $44 \%(P<0,001)$. La pression à exercer sur le trayon pour ouvrir le canal est plus faible : 10,9 contre $17,2 \mathrm{kPa}(P<0,001)$. D'une façon moins marquée, il apparaît aussi que l'ouverture du canal du trayon se fait plus complètement le matin que le soir puisque la quantité de lait recueillie sous succion constante est accrue de $20 \%(P<0,01)$.

\section{Corrélations entre les paramètres qui caractérisent la traite (tableau II)}

La bibliographie fait état de nombreuses estimations des corrélations phénotypiques calculées entre les divers critères de traite : productions, temps, débits (revue 
de Szeremeta, 1987). Elles sont établies dans des conditions a priori différentes des nôtres. En général, elles concernent la mamelle entière et les temps sont mesurés par chronométrage manuel, ce qui entraî̉ne une grande variabilité de l'appréciation de la fin réelle de la traite. Or, dans notre cas, chaque quartier est trait séparément tandis que la cinétique d'émission du lait est enregistrée automatiquement. Malgré ces différences, nous constatons que nos résultats (tableau II) sont globablement en accord avec les valeurs moyennes rapportées par Szeremeta (1987) et, par ailleurs, classiquement admises. La seule différence notable concerne le volume d'égouttage. Dans notre cas, il n'est corrélé avec aucun des autres critères bien qu'il augmente de façon non significative avec le débit maximal $(r=0,16)$. À l'inverse, Szeremeta mentionne une liaison faible mais négative $(r=$ $-0,21$, selon Politiek, 1961). La contradiction éventuelle n'est qu'apparente puisqu'on sait que le volume d'égouttage dépend beaucoup de l'appréciation de la fin de traite et de la machine à traire dont la conception a beaucoup changé depuis 1961.

\section{Corrélations entre les propriétés physiques des trayons (tableau II)}

Il se dégage des résultats que lẹs corrélations significatives entre les différents critères sont toujours très cohérentes. Elles permettent d'identifier les caractéristiques du trayon susceptibles d'influencer l'écoulement du lait pendant la traite et donc de mieux cerner la notion d'aptitude à la traite.

Les corrélations entre les caractéristiques dimensionnelles sont toutes significatives : l'épaisseur des parois et des tissus de l'extrémité du trayon diminue avec le diamètre $(P<0,001)$.
Les différents critères utilisés pour évaluer la résistance du canal du trayon à l'ouverture sont tous significativement corrélés entre eux : succion ou pression pour avoir un écoulement du lait, quantité de lait recueillie en $20 \mathrm{~s}$ sous succion constante $(P<0,01$ ou $P<0,001)$.

Globalement, il apparaît que les trayons les plus faciles à traire ont un faible diamètre et des parois peu épaisses. Toutes les corrélations tendent à démontrer que le canal du trayon est entouré de tissus peu volumineux et élastiques.

On observe notamment que, lorsque l'épaisseur des tissus de l'extrémité du trayon augmente, la succion nécessaire pour ouvrir le canal $(P<0,01)$ ainsi que la pression augmentent $(P<0,001)$, tandis que la quantité de lait recueilli en $20 \mathrm{~s}$ sous succion constante diminue $(P<$ $0,01)$. Ces observations sont compatibles avec ce qui est généralement admis (revue de Labussière et Richard, 1965) et avec les conclusions auxquelles nous étions parvenus en étudiant la chèvre dans un contexte expérimental similaire (Le Du et Benmederbel, 1984).

\section{Corrélations entre les caractéristiques de traite et les propriétés physiques des trayons (tableau II)}

Les rares corrélations significatives où interviennent les productions laitières ne nous semblent pas interprétables. II s'agit notamment des corrélations positives entre les productions de lait machine ou total et l'épaisseur des parois du trayon ( $r$ entre 0,24 et 0,28 ) ou des tissus de son extrémité ( $r$ entre 0,39 et 0,43 ). Notsuki et Goto (1967b) avaient déjà relevé que la production totale était corrélée positivement non seulement avec l'épaisseur des parois du trayon $(r=+0,34)$ mais aussi avec son diamètre $(r=+0,49)$, ce qui confirme Ra- 


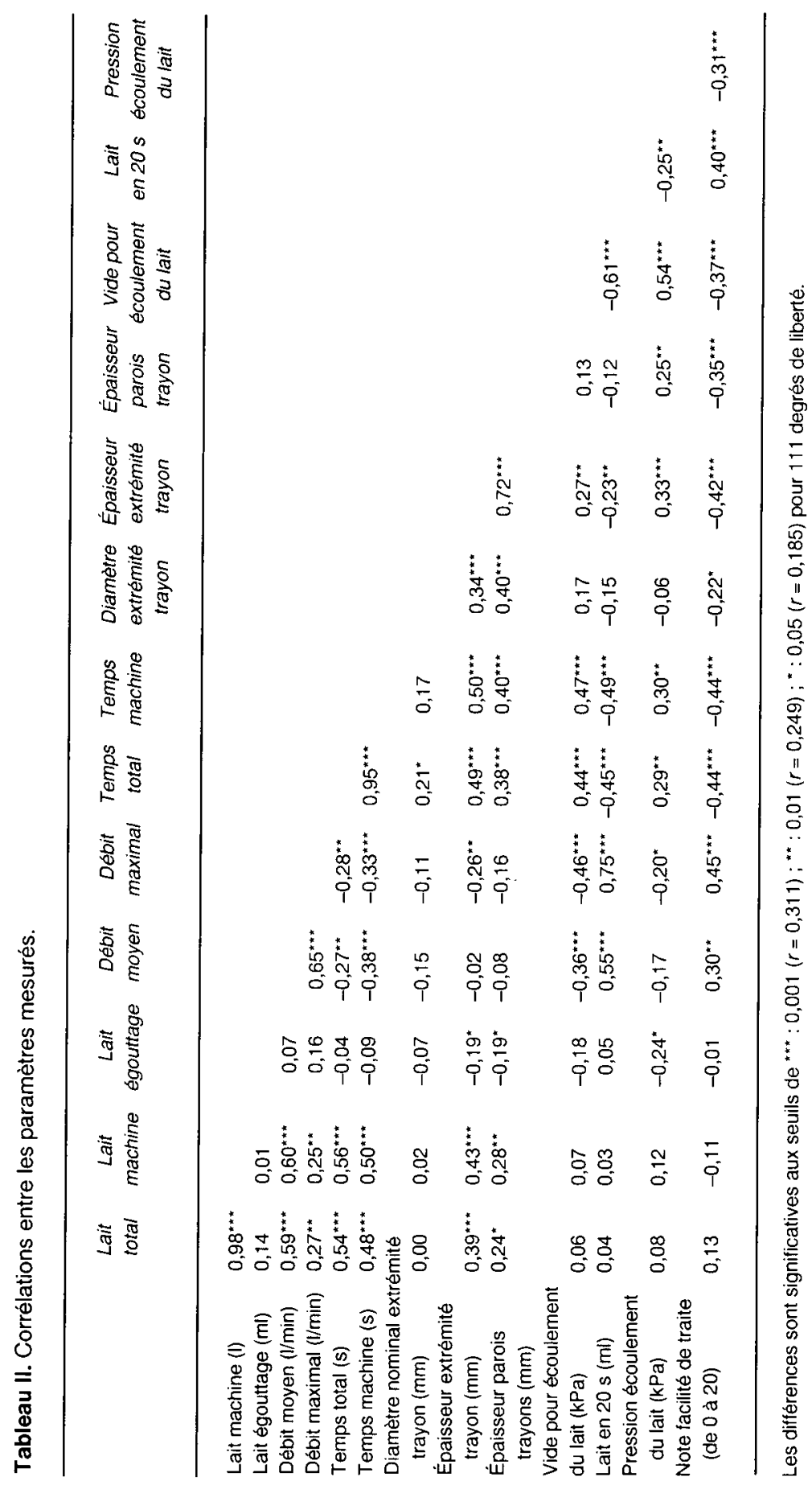


thore (1977) $(r=+0,35)$, alors que sur ce dernier point nous trouvons une corrélation pratiquement nulle.

D'une façon générale, les liaisons phénotypiques brutes entre les dimensions du trayon et les caractéristiques de traite qui sont rapportées dans la bibliographie sont souvent contradictoires. D'une part, les caractéristiques de traite dépendent beaucoup de la machine à traire utilisée, et notamment du type de manchon trayeur (Le Du et Richard, 1977 ; Le Du et Taverna, 1989). D'autre part, l'adaptation du manchon trayeur aux dimensions du trayon étant plus ou moins parfaite, il est probable que les différences observées entre les caractéristiques de traite des trayons peuvent être soit masquées, soit amplifiées.

Le modèle mathématique de trayon élaboré par Balthazar et Scott en 1977 nous semble susceptible de pallier les difficultés rencontrées pour interpréter les mesures directement sur les animaux. En théorie, selon ce modèle, "le débit du lait augmente avec un trayon ayant un grand diamètre extérieur et des parois minces". Qualitativement, nous confirmons ces hypothèses. Les corrélations entre les temps de traite et l'épaisseur des parois (environ 0,40 ), ou mieux l'épaisseur de l'extrémité du trayon (environ 0,50), sont les plus élevées. En revanche, elles sont nettement moins élevées lorsqu'on considère le débit ( $r$ entre 0 , et 0,26 ) au lieu du temps de traite.

Finalement, ces résultats confirment la validité du modèle de Balthazar et Scott qui prévoit que, pour un trayon d'un diamètre extérieur donné, la section du canal est d'autant plus grande que l'épaisseur des tissus qui l'entourent est faible.

Les corrélations entre les critères qui permettent d'évaluer plus directement l'aptitude du canal du trayon à s'ouvrir par pression ou par succion s'interprètent très logiquement dans le contexte de nos ob- servations précédentes. Dans tous les cas, on note une diminution des temps de traite et un accroissement des débits lorsque les mesures montrent que le canal du trayon s'ouvre facilement sous l'effet d'une pression ou d'une succion.

Le débit et le temps de traite sont des critères couramment retenus pour la sélection sur la facilité de traite. Toutefois, dans les conditions d'élevage en fermes, les mesures sont longues à effectuer et d'une faible précision car la fin de traite est difficile à apprécier. Les généticiens admettent donc que le débit maximal représente la mesure plus objective de l'aptitude à la traite (Celerier et al, 1972, 1975 ; Szeremeta, 1987).

Le critère le plus étroitement corrélé avec le débit maximal est la quantité de lait recueillie en $20 \mathrm{~s}$ sous succion constante. La corrélation $(r=+0,75)$ est voisine de celle qui a été déterminée par Notsuki et Goto (1967b) $(r=+0,80)$. La corrélation entre la mesure du vide permettant l'écoulement du lait et le débit maximal ( $r=$ $-0,46$ ) est, en revanche, plus faible que pour Notsuki et Goto (1987b) ( $r=-0,81$ ). La mesure de la pression permettant l'écoulement du lait n'est que faiblement corrélée avec le débit maximal $(r=-0,20)$ alors que les auteurs japonais mentionnent des valeurs élevées voisines de $-0,70$ (Naito et al, 1965b ; Notsuki et Goto, 1967b).

Les temps de traite (machine et total) sont toujours corrélés de façon significative avec les critères permettant d'évaluer par succion ou par pression la résistance à l'ouverture du canal du trayon.

\section{Corrélations avec la note de facilité de traite (tableau II)}

Le moyen le moins coûteux de contrôler la vitesse de traite repose sur l'appréciation 
des caractéristiques de traite par l'éleveur lui-même. Dans de nombreux pays, cette méthode dite subjective est utilisée notamment pour détecter les taureaux ayant des proportions anormalement élevées de filles difficiles à traire (Szeremeta, 1987). Dans tous les cas, on demande à l'éleveur d'apprécier l'aptitude à la traite en classant chaque animal selon un barème dont le plus simple comprend seulement 3 classes telles que : difficile à traire, normale ou facile à traire. Évidemment, la question se pose de savoir quel est le caractère objectivement désigné par l'appellation "facile à traire».

Pour notre étude, après consultation des 5 trayeurs qui devaient évaluer l'aptitude à la traite de nos vaches, nous avons choisi de libeller les 3 classes de la façon suivante : facile à traire (20), normale (10) et longue à traire (0). L'examen des résultats (tableau II) montre que :

- la note est particulièrement bien corrélée avec le temps de traite machine $(r=$ $-0,44)$ et total $(r=-0,44)$, ainsi qu'avec le débit machine $(r=+0,30)$ et maximal $(r=$ $+0,45$ ). II est surprenant de constater que la caractéristique de traite estimée avec le plus de précision est le débit maximal. En effet, d'une part, il est considéré comme le critère le plus pertinent pour évaluer la vitesse de traite, mais en contrepartie, sa mesure est difficile puisqu'elle nécessite d'utiliser un équipement spécial d'enregistrement de la cinétique d'émission du lait ;

- la note n'est pas corrélée avec les productions laitières qui sont pourtant fortement corrélées avec les temps : $r=+0,50$ en moyenne. Cela montre que le nombre de 4 faisceaux par trayeur est suffisamment élevé pour ne pas avoir d'incidence sur la productivité horaire. En d'autres termes, dans nos stalles en tandem où la traite de chaque vache est relativement individualisée, l'animal peut occuper sa stalle pendant tout le temps nécessaire à une traite complète sans que cela soit ressenti comme une gêne par le trayeur puisqu'il dispose d'un nombre suffisant de faisceaux. En revanche, dans une salle de traite en épi où les animaux sont traits par lots, la productivité peut être limitée par le temps de traite de la vache la plus longue à traire du lot. Les résultats d'enquêtes où le trayeur doit noter ses vaches d'une façon relativement subjective pourraient dépendre des facteurs de milieu liés à la conception de l'installation de traite;

- la note n'est pas corrélée au volume d'égouttage. Or, au même titre que la préparation du pis avant la traite, l'égouttage représente une intervention manuelle qui a une incidence directe sur la productivité. II est d'ailleurs courant que le trayeur qualifie une vache de "dure à traire" parce qu'elle «retient son lait», ce qui signifie implicitement qu'un égouttage prolongé est nécessaire pour vider complètement le pis. L'absence de corrélation peut s'expliquer par le fait que, dans nos conditions de travail, les trayeurs ont pour consigne d'effectuer un égouttage standardisé de durée limitée qui vise à contrôler que la vidange du pis est satisfaisante sans chercher à extraire la quasi-totalité du lait présent ;

- les corrélations entre la note et les caractéristiques du trayon (autres que le diamètre) sont hautement significatives et s'interprètent logiquement dans le contexte de nos conclusions précédentes.

\section{CONCLUSION}

Nos résultats permettent de mieux cerner les facteurs qui conditionnent l'aptitude à la traite en précisant notamment l'incidence des caractéristiques du trayon.

En général, on admet que c'est le déséquilibre antéro-postérieur de la mamelle qui explique que les temps de traite et les débits d'écoulement du lait sont plus éle- 
vés pour les quartiers arrière. Or, dans nos conditions expérimentales, la comparaison avec les résultats obtenus aux traites du matin ( $64 \%$ de la production journalière) et du soir, espacées de $15 \mathrm{~h}$, montre que la disparité des productions de lait n'explique pas totalement les différences de temps de traite et de débits observés entre les quartiers avant et arrière. Comme certains auteurs antérieurement (Balthazar et Scott, 1977 ; Rathore et Sheldrake, 1977), nous confirmons que les trayons arrière se traient plus facilement que ceux de l'avant. D'une part, la pression ainsi que la succion à exercer pour ouvrir le canal du trayon et obtenir un début d'écoulement du lait sont significativement plus faibles. D'autre part, la section du canal du trayon est plus extensible puisque la quantité de lait recueillie en $20 \mathrm{~s}$ avec le vacuomètre est significativement plus élevée que pour les trayons avant. Ce constat pourrait expliquer que les infections mammaires sont plus fréquentes dans les quartiers arrière (FIL, 1987) où les micro-organismes pathogènes pourraient pénétrer plus facilement du fait de la structure plus lâche du canal du trayon (Grindal et Hillerton, 1991 ; Grindal et al, 1991). Le vacuomètre est un équipement très simple qui pourrait permettre de vérifier, dans les conditions rencontrées en fermes, la validité de cette hypothèse.

Globalement, il apparaît que l'écoulement du lait est facilité lorsque le trayon a des parois peu épaisses et un canal terminal entouré de tissus peu volumineux et élastiques. Ainsi, par exemple, la corrélation entre le temps total de traite et l'épaisseur des tissus de l'extrémité du trayon atteint $0,50(P<0,001)$.

Dans la bibliographie, les liaisons phénotypiques brutes entre les propriétés des trayons et les caractéristiques de traite sont souvent contradictoires. En effet, les caractéristiques de traite dépendent beaucoup de la machine à traire utilisée. II im- porte de souligner que, dans nos conditions expérimentales, la cinétique d'émission du lait est enregistrée automatiquement pour chaque quartier. Les temps et les débits sont donc mesurés avec une grande précision, ce qui n'est pas le cas avec les méthodes qui reposent sur un chronométrage manuel.

Dans ce contexte, nous observons dans tous les cas une diminution des temps de traite et un accroissement des débits lorsque les mesures effectuées avec le garrot et le vacuomètre montrent que le canal du trayon s'ouvre facilement. Le débit maximal, qui est pour les généticiens la mesure la plus objective de l'aptitude à la traite (Celerier et al, 1972, 1975 ; Szeremeta, 1987), est notamment bien corrélé avec la quantité de lait recueillie en 20 s sous succion constante avec le vacuomètre : $r=$ $+0,75$. Cela confirme parfaitement les travaux antérieurs : $r=+0,80$ pour Notsuki et Goto (1967b). Dans les conditions d'élevage en ferme, la mesure des débits est longue et d'une faible précision. À l'inverse, le vacuomètre est un appareil facile à manier avec lequel les mesures sont effectuées rapidement : 20 à 30 vaches par heure. II pourrait éventuellement permettre au généticien d'apprécier rapidement l'aptitude à la traite, indépendamment des effets du milieu. Les modalités d'emploi optimales du vacuomètre, ainsi que les liaisons phénotypiques (et génotypiques) avec la facilité de traite, demanderaient à être précisées.

L'évaluation de la facilité de traite par notation subjective donne des résultats très satisfaisants. La note est significativement corrélée avec tous les paramètres qui caractérisent le trayon et les conditions de traite, à l'exception des productions de lait (total, machine ou égouttage). II est suprenant de constater que le paramètre estimé avec le plus de précision est le débit maximal dont la mesure est pourtant la plus difficile $(r=0,45 ; P<0,001)$. II 
convient de rappeler que nous avons travaillé dans des conditions qui ne sont pas celles que I'on rencontre en fermes. II s'agit d'un troupeau expérimental, et la note finale représente la moyenne des notes attribuées par 5 techniciens expérimentés chargés de la traite.

\section{REMERCIEMENTS}

Nous exprimons notre reconnaissance au personnel de la ferme expérimentale du Rheu pour la collaboration apportée à la réalisation de ce travail.

\section{RÉFÉRENCES}

Andreae $U$ (1958) Measurement of milk flow from cow's udders in investigating ease of milking. Zuechtungskunde 27, 238-244

Balthazar JA, Scott NR (1977) Deformations of the dairy cow's teat by finite element analysis. ASAE. Paper $n^{\circ} 77,3543$

Barczynski G (1960) Ein experimenteller Beitrag zuden anatomisch funktionellen Grundlagen der Melkugenschaften des Rindes. Inaug Diss Vet, Hannover, 48

Baxter ES, Clarke PM, Dodd FH, Foot AS (1950) Factors affecting the rate of machine milking. J Dairy Res 17 (2), 117-127

Benmederbel B (1980) Incidence du degré de remplissage de la mamelle sur les propriétés physiques du trayon chez la vache. Rapport de DEA, Labo Recherches Traite, INRA, Rennes

Celerier B, Duplan JM, Szeremeta A (1972) Le contrôle des caractéristiques de traite par la méthode dite "Quatre quartiers". Son importance et ses résultats en France. Élevage \& Insémination, juillet 1972, 130, 1-30

Celerier B, Duplan JM, Szeremeta A (1975) Le contrôle des caractéristiques de traite (suite). La précision des estimations de la valeur génétique pour l'aptitude à la traite, facteur de progrès rapide. Élevage \& Insémination, novembre $1975,150,1-14$

Colleau JJ (1971) Intérêt du temps total de traite pour la sélection des vaches sur la vi- tesse de traite. II. La sélection des femelles. Ann Genet Sel Anim 3, 315-330

Dodd FH, Griffin TK (1979) Milking routines in "machine milking" (Thiel and Dood) NIRD Reading, 179-199

FIL (1987) Machine milking and mastitis. In: Bull IDF 215, Ed IDF, Brussels

Grindal RJ, Hillerton JE (1991) Influence of milk flow rate on new intrammary infection in dairy cows. J Dairy Res 58, 263-268

Grindal RJ, Walton AW, Hillerton JE (1991) Influence on milk flow rate and streak canal length on new intramammary infection in dairy cows. J Dairy Res 58, 383-388

Hamann J, Mein GA (1990) Measurement of machine induced changes in thickness of the bovine teat. J Dairy Res 57, 495-505

Ipsen EJ (1975) La traite. Den KGL Veterinaer og Landbohojskole. Ed DSR Forlag, Copenhague, Danemark (en danois)

Johansson I (1957) Investigations into the variation in shape of the udder and teats of cows. $Z$ Tierz Zuechtungsbiol 70, 223-270 (en allemand)

Johansson I, Malven P (1960) The influence of yield, udder pressure, size of teats and of the teat orifice on the rate of milking. $Z$ Tierz Zuechtungsbiol 74, 1-3 (en allemand)

Karavaev Yu S (1966) Theoretical prerequisites of assessment of milking resistance of cow's teats. Izv Timiryazev S Khoz Akad 6, 229231 (en russe)

Kartashov LP (1973) Determination of teat sphincter tonus in lactating cows. Zhivotnovodstvo 12, 48-49 (DSA, 1974, 34, 2607)

Kuhne M (1959) Strukturverhältnisse im Bereich des Zitzenstrichkanals und ihre Beziehungen zur Melkbarkeit. Inaug Vet Diss, Hannover, 31

Krychowski T (1972) La sélection sur la vitesse de traite dans l'espèce bovine : comparaison de deux méthodes d'appréciation de la valeur des taureaux. Inst Natl Agron ParisGrignon (ronéoté)

Labussière $J(1972)$ Extensiomètre de trayon. Brevet Français, $\mathrm{n}^{\circ} 2,102475$

Labussière J, Martinet $J(1964)$ Description de deux appareils permettant le contrôle automatique des débits de lait au cours de la traite à la machine. Premiers résultats obtenus chez la brebis. Ann Zootech 13, 199-212 
Labussière J, Richard P (1965) La traite mécanique : aspects anatomiques, physiologiques et technologiques. Mise au point bibliographique. Ann Zootech 14, 63-126

Le Du J, Richard J (1977) Influence du manchon-trayeur sur les caractéristiques de traite des vaches de race Française Frisonne Pie Noire. Ann Zootech 26, 503-512

Le Du J, Benmederbel B (1984) Aptitude des chèvres de race Saanen à la traite mécanique. Relations avec les caractéristiques physiques du trayon. Ann Zootech 33, 375-384

Le Du J, Taverna M (1989) Manchons trayeurs pour vaches : effets du diamètre et de l'élasticité du corps sur les caractéristiques de traite et sur quelques mesures de l'extrémité du trayon. Ann Zootech 38, 19-28

Loppnow H (1959) Influence of teat structure on milking ability. Dtsch Tieraerztl Wochenschr $66,88-97$ (en allemand)

McDonald JS (1968a) Radiographic method for anatomic study of the teat canal: changes with lactation age. Am $J$ Vet Res 29, 12071210

McDonald JS (1968b) Radiographic method for anatomic study of the teat canal: observations on 22 lactating dairy cows. Am $J$ Vet Res 29, 1315-1319

McDonald JS (1975) Radiographic method for anatomic study of the teat canal: changes between milking periods. Am $J$ Vet Res 36 , 1241-1242

Mein GA, Williams DM (1984) Liner massage and teat conditions. Proc 23rd in Meeting Natl Mastitits Council, Kansas City, USA (Natl Mast Council, ed). Washington DC, USA, 4-18

Naito $M$, Shoda $Y$, Kobayashi $H$, Fukushima $T$ (1965a) Changes of milking characteristics and teat characters during lactation in dairy goats. Jpn J Zootech Sci 36 (5), 170-176 (en japonais)

Naito $M$, Shoda $Y$, Kobayashi $H$, Fukushima $Y$, Nomura S (1965b) Relationship between various characters of teats and milking characteristics in Holstein-Friesian cows. Jpn J Zootech Sci 36 (11), 496-505 (en japonais)

Naude RT, Smith A (1964) The relationship between teat conformation and milkability of the cow. Proc S Afr Soc Anim Prod 3, 101-103 (en afrikaans)
Notsuki I, Goto T (1967a) Studies in milkability of dairy cows in machine milking. 1. Some characters of teats in dairy cows. Bull Natl Inst Ind Chiba 13, 41-48 (en japonais)

Notsuki I, Goto T (1967b) Studies of milkability of dairy cows in machine milking. 2. Relationship between characters of teat and milkability in dairy cows. Bull Natl Inst Ind Chiba 13, 49-58 (en japonais)

O'Shea J, O'Callaghan E (1980) Milking characteristics of 30 cluster types. In: Experiments on milking machine components at Moorepark 1976-1979 (O'Shea, O'Callaghan, eds) An Foras Taluntais, Dublin, 170-191

Ovesen EB (1970) Étude des capacités morphologiques de la mamelle chez les vaches en première lactation. Thèse, Royal Veterinary College (en danois) (cité par Ipsen EJ, 1975)

Ovesen EB (1972) Milking ability in relation to size and shape of teats. Anim Prod 15, 251257

Politiek RD (1961) Observations sur l'analyse de la facilité de traite, sur sa variabilité chez les vaches ainsi que sur l'héritabilité de ce caractère. Extrait du $B T I n^{\circ} 166$, janvier 1962 (cite par Szeremeta, 1987).

Rathore AK (1977) Teat diameter gradient associated with milk yield and somatic cell count in British Friesian cows. Anim Prod 24, 401406

Rathore AK, Sheldrake RF (1977) Teat orifice stretchability associated with teat diameter gradient and milk yield in lactating cows. Anim Prod 24, 215-220

Sandvik $O$ (1957) The influence of size and shape of udder and teats in milk yield and milking characters in cows. Meld Nor Landbrukshogsk 36 (8), 1-8 (en norvégien)

Schmitten F, Klüsserath D (1968) Mikability assessment by ultrasonic measurements. Tierzucht 20 (2), 34-35 (en allemand)

Skallcup JM, Rakes JM, Ford GL (1963) Relationship between milk flow and anatomical characteristics of the udder. J Dairy Sci 46, 624-625

Szeremeta A (1987) Le contrôle de l'aptitude à la traite chez les bovins laitiers. Place et perspectives de la "méthode complète". Thèse de doctorat, ITEB, Paris

Towsend JS (1969) A mathematical modeling approach to the study of the mechanics of 
milking machine operation. Unpublished Ph D thesis, Cornell University, Ithaca, New York

Valdmann $E$ (1959) Effect of the teat sphincter and the milking rate of machine milked cows. Sborn Nachn Trud Estonsk nauchno-issled. Zhivotnov'd Vet 3, 39-51 (en estonien) (DSA $n^{\circ} 25,1306$ )

Wilke G (1959) Milkability, its dependence on udder shape and its effect on lactation curve. $Z$ Tierz Zuechtungsbiol 74, 48-86 (en allemand)

Williams DM, Mein GA (1982) Review: physical and physiological factors affecting milk flow rate from the bovine teat during machine milking. In: Proc conf on dairy production from pasture (KL Macmillan, VK Taufa, eds) Hamilton, Nouvelle Zélande, 42-74

Zecconi A, Hamann J, Bronzo V, Ruffo G (1992) Machine induced teat tissue reactions and infection risk in a dairy herd free from contagious mastitis pathogens. I Dairy Res 59, 265-271

Zhukov AE (1971) Teat sphincter tonus in cows and machine milking. Vestn S'kh Nauki Mosc 16 (12), 20-24 (en russe) 